\title{
Hydrogen electrosorption properties of electrodeposited Pd-Ir alloys
}

\author{
K. Hubkowska ${ }^{1}$ (D) M. Pająk $k^{1,2} \cdot$ A. Czerwiński ${ }^{1,2}$ \\ Received: 24 September 2020 / Revised: 12 April 2021 / Accepted: 7 May 2021 / Published online: 14 May 2021 \\ (C) The Author(s) 2021
}

\begin{abstract}
The study of the hydrogen sorption process in metals/alloys of different forms is crucial for developing the fields of catalysis and energy storage. The objective of this research was to examine basic hydrogen electrosorption properties of Pd-Ir alloy thin films obtained through electrodeposition. Pd-Ir alloys, containing more than $87 \%$ at. Pd, were successfully potentiostatically electrodeposited from aqueous baths containing $\mathrm{PdCl}_{2}$ and $\mathrm{IrCl}_{3}$. X-ray diffractometry confirmed the Pd-Ir alloy formation and homogeneity of the deposits. The hydrogen electrochemical absorption was carried out with the use of cyclic voltammetry and chronoamperometry. The values of $H /(\mathrm{Pd}+\mathrm{Ir})$ for Pd-Ir alloys containing less than $3 \%$ at. Ir are similar, comparable to Pd. The further increase of Ir content results in the abrupt drop of hydrogen absorbing capacity. The linear decrease of the potential of $\alpha \rightarrow$ $\beta$ phase transition versus Pd content is observed, confirming classification of the Pd-Ir alloys to the group of the contracted alloys.
\end{abstract}

Keywords Hydrogen sorption · Palladium-iridium alloys $\cdot$ Contracted alloys $\cdot \alpha \rightarrow \beta$ phase transition $\cdot \mathrm{Pd}-\mathrm{Ir}-\mathrm{H}$ system

\section{Introduction}

The process of hydrogen electrosorption has been examined in details in $\mathrm{Pd}$ [1-6], whereas hydrogen absorbing properties of some of Pd-based binary alloys are still unknown. The literature contains studies on a variety of Pd-M-H systems with different $\mathrm{M}$ elements such as $\mathrm{Rh}, \mathrm{Ru}, \mathrm{Pt}, \mathrm{Au}, \mathrm{Ni}, \mathrm{Cu}$, and Ag [7-18] but there is no information about Pd-Ir alloys in the aspect of hydrogen electrosorption.

The phase diagram of Pd-Ir system shows a large miscibility gap in the solid state. Thus, in the Pd-Ir systems at thermodynamic equilibrium two phases may be present in the solid state, i.e., Ir solid solution in Pd for Pd-rich alloys and a Pd solid solution in Ir for Ir-rich alloys [19]. The lattice constant of homogeneous Pd-Ir alloys is smaller than that of pure Pd and exhibits negative deviations from Vegard's law [20].

This article is dedicated to memory of Professor Roberto Marassi, great electrochemist and my friend with whom we cooperated scientifically for almost 40 years. - Andrzej Czerwiński

K. Hubkowska

khubkowska@chem.uw.edu.pl

1 Faculty of Chemistry, University of Warsaw, Pasteura1, 02-093 Warsaw, Poland

2 Biological and Chemical Research Centre, University of Warsaw, Żwirki i Wigury 101, 02-089 Warsaw, Poland
Since with the Pd alloying with Ir the lattice constant decreases, Pd-Ir alloys can be classified to the group of contracted alloys. Generally, classification to the group of expanded or contracted alloys is reflected on the course of the hydrogen sorption isotherms. In the case of contracted alloys hydrogen sorption isotherms are shifted into higher values of hydrogen pressures or respectively into lower potentials (for hydrogen electrosorption). Thus, it is expected that alloying Pd with Ir will result in the described behavior.

$\mathrm{Pd}$ and Ir belong to the platinum group metals; however, their interaction with hydrogen is different. Pd is able to adsorb and absorb hydrogen, whereas in the case of Ir only hydrogen adsorption is possible [21, 22]. Hydrogen absorption in Pd-Ir alloys was studied only by a few authors. LaPrade et al. [23] studied hydrogen absorption from the gas phase in the bulk, annealed Pd-Ir alloys. They found that the hydride phase is formed for the alloys containing less than approximately $9 \%$ of Ir. The authors also stated that the lattice constant expansion after hydrogen dissolution in Pd-Ir alloys is smaller than for pure Pd and decreases with increasing amount of Ir. Akamaru et al. [24], Zlotea et al. [25], Malouche et al. [26], and Kobayashi et al [27] studied the hydrogen sorption properties of Pd-Ir nanoalloys. The authors noticed that in PdIr nanoalloys the ability for hydrogen absorption occurs for a much wider range of compositions than in the bulk alloys. All the cited results refer to hydrogen sorption from the gas phase and they could differ from those obtained through hydrogen electrosorption from electrolytes. 
According to the electronic effect [28] the maximum amount of absorbed hydrogen in Pd alloys with a nonabsorbing metal (e.g., $\mathrm{Au}, \mathrm{Ag}, \mathrm{Pt}$ ) decreases with the increase of the content of alloying metal. It can be explained by the band model. Since the gaps in the Pd dband are partially filled by the alloying metal electrons, less gaps remain for the electrons from absorbed hydrogen. However, in Pd alloys with metals characterized by an increased number of free electronic states in d-band below Pd Fermi level, such as $\mathrm{Rh}$ and $\mathrm{Ru}$, in a certain composition range greater hydrogen capacity is observed $[9,11,12,28]$. Since Ir has the electronic structure similar to $\mathrm{Rh}$, it can be expected that Pd-Ir alloys will possess hydrogen absorption properties close to those of Pd-Rh. These predictions were not confirmed by the results published by LaPrade et al. [23], Akamaru et al. [24], and Kobayashi et al. [27]. However, they provided data only for a few alloy compositions, with the Ir content lower than $3 \%$. The previous studies $[11,12]$ on the electrodeposited Pd-Ru alloys have revealed that hydrogen absorption properties may be very sensitive to bulk composition and already $0.5-1 \%$ substitution by Ru has led to distinctly increased hydrogen absorption capacity, while after further $\mathrm{Ru}$ addition the alloy ability to absorb hydrogen drastically decreased. Therefore, more detailed investigations on hydrogen absorption into Pd-Ir alloys with wider composition range are necessary, in particular for alloys with very small Ir contents (below 3\%).

The discussion above, which indicates the possibility of enhanced hydrogen absorption capacities of Pd-Ir alloys compared to Pd, were the main motivation to perform the studies under this system. Despite the relatively high costs of noble metals Pd-Ir system is still investigated in view of different processes such as electrocatalytic activity towards oxygen reduction [29], hydrogen oxidation [30], hydrogen evolution reaction [31], and hydrogenation reaction [32]. Moreover, the cost of thin (approximately $1 \mu \mathrm{m}$ ) Pd-based films is significantly lower than the cost of the bulk materials (for Pd-Ir thin film electrode containing approximately $13 \%$ at. Ir it is approximately $0.1 € / \mathrm{cm}^{2}$ ).

The main aim of this preliminary study was to present the effectiveness of electrodeposition method in obtaining thin films (approximately $1 \mu \mathrm{m}$ ) of Pd-rich Pd-Ir alloys and express the potential of this alloy as a hydrogen-absorbing material.

In the literature there is a lack of information on the electrodeposited Pd-Ir alloys and their hydrogen sorption properties obtained electrochemically. Therefore, in this paper the results of hydrogen electrosorption properties of electrodeposited Pd-Ir alloys are presented. Moreover, the new insight into the hydrogen capacity of the bulk, electrodeposited Pd-Ir alloys, and $\alpha \rightarrow \beta$ phase transition potential variation with the $\mathrm{Pd}$ content is discussed.

\section{Experimental}

\section{Materials}

Palladium(II) chloride was purchased from Sigma-Aldrich and iridium(III) chloride hydrate was obtained from Alfa Aesar. Au wire (99.99\%; diameter: $0.5 \mathrm{~mm}$ ) and Au plate (99.99\%; thickness: $0.12 \mathrm{~mm}$ ) were supplied from MintMetals (Poland). The $0.5 \mathrm{M} \mathrm{H}_{2} \mathrm{SO}_{4}$ water solution was prepared from analytical grade reagent (Avantor Performance Materials Poland S.A.) and water purified in the Hydrolab and the Millipore system (the conductivity of water after purification: approximately $0.054 \mu \mathrm{Scm}^{-1}$ ).

\section{Pd-Ir alloy preparation}

Pd-Ir alloys were deposited electrochemically on the Au wire (used for electrochemical measurements and SEM analysis) or Au plate (used for XRD and SEM analysis) from the mixtures of water solutions: $0.11 \mathrm{M} \mathrm{PdCl}_{2}$ in $1 \mathrm{M} \mathrm{HCl}$ and $0.5 \mathrm{M} \mathrm{IrCl}_{3}$. The galvanic baths used for electrodeposition contain the following concentrations of $\mathrm{Pd}$ (II) ions: $0.03-0.07 \mathrm{M}$ and $\operatorname{Ir}(\mathrm{III})$ ions: $0.28-0.42 \mathrm{M}$. Variety of alloy compositions were obtained by the use of different concentration of the ions in the baths and the application of different values of electrodeposition potentials $(0.38-0.18 \mathrm{~V})$. The thickness of the electrodeposits calculated from atomic absorption spectroscopy measurements (AAS) was in the range of approximately $0.8-1.1 \mu \mathrm{m}$ and the deposition efficiency was in the range of approximately $86-97 \%$.

\section{Physicochemical characterization of Pd-Ir alloys}

The bulk compositions of the Pd-Ir alloys were determined with the use of AAS (Thermo-Scientific 3300, USA) and energy dispersive X-ray spectroscopy (EDS; Quantax 400, Bruker, USA). In the latter measurement $\mathrm{X}$-ray excitation with electron beam energy of $10-15 \mathrm{keV}$ and spectrum acquisition time of approximately 100-120 s were applied. For energy calibration, X-ray signals obtained from pure $\mathrm{Cu}$ were utilized.

The surface morphology of alloys were examined with a Merlin scanning electron microscope (SEM, Zeiss, Germany).

X-ray diffraction (XRD) patterns were collected by D5000 (Bruker AXS) diffractometer with an $\mathrm{Cu}$ anode $(40 \mathrm{~mA}, 40$ $\mathrm{kV})$ and a Ni filter (1:20). The detector with 192 measuring LEDs (A Lynx Eye, Bruker AXS) was used.

\section{Electrochemical characterization of Pd-Ir alloys}

The electrochemical characterization of Pd-Ir alloys was carried out in the water solution of $0.5 \mathrm{M} \mathrm{H}_{2} \mathrm{SO}_{4}$ in the threeelectrode system: Pd-Ir alloy electrode was a working 
electrode, $\mathrm{Pt}$ gauze was an auxiliary electrode, and $\mathrm{Hg}\left|\mathrm{Hg}_{2} \mathrm{SO}_{4}\right| 0.5 \mathrm{M} \mathrm{SO}_{4}{ }^{2-}$ was a reference electrode. Cyclic voltammetry $(\mathrm{CV})$ and chronoamperometry $(\mathrm{CA})$ were used in electrochemical measurements. All potential values are expressed versus RHE.

The electrodes were saturated with hydrogen at fixed potential values (from approximately $0.18 \mathrm{~V}$ to approximately $-0.12 \mathrm{~V}$ ) for sufficient time ensuring full hydrogenation. Then the absorbed hydrogen was oxidized with the use of CV (10 $\mathrm{mVs}^{-1}$ ) starting form the value of saturation potential (from approximately $0.18 \mathrm{~V}$ to approximately $-0.12 \mathrm{~V}$ ) to the value of approximately $0.38 \mathrm{~V}$. The scan rate of $10 \mathrm{mVs}^{-1}$ was chosen to be the most optimal after the preliminary experiment involving the influence of the scan rate on the hydrogen oxidation charge.

The charge was calculated by the integration of hydrogen oxidation signal $(Q)$ and then recalculated into $H /(\mathrm{Pd}+\mathrm{Ir})$, according to the formula: $H /(\mathrm{Pd}+\mathrm{Ir})=Q /(\mathrm{F} \cdot \mathrm{n})$, where $\mathrm{F}$ is a Faraday's constant and $n$ expressed sum of the number of Pd and Ir moles (AAS). In this manner approximately 26-30 points were collected to create hydrogen electrosorption (absorption) isotherm (HAI: $H /(\mathrm{Pd}+\mathrm{Ir})$ vs. $E_{\text {abs }}$ ). The $\alpha \rightarrow \beta$ phase transition potentials $\left(E_{\alpha \rightarrow \beta}\right)$ were determined from the inflection points at the HAIs (from the area of the abrupt increase of hydrogen content). The maximum hydrogen capacities (maximum values of $H /(\mathrm{Pd}+\mathrm{Ir})$ ) were determined from the area of $\beta$-phase plateau at HAI. Under described experimental conditions adsorbed hydrogen has only negligible influence on the value of $H / \mathrm{M}$ ratio.

\section{Results and discussion}

\section{Structural and morphological analysis of Pd-Ir alloys}

Thin films of electrodeposited Pd-Ir alloys were subjected to preliminary physicochemical characterization involving SEM, EDS, and XRD analysis.

SEM image in Fig. 1a presents the morphology of the fresh Pd-Ir (approximately 97\% Pd, EDS) electrode after the electrodeposition process. It can be noticed that the thin film electrodeposited on a gold substrate is compact and not cracked. After hydrogen electrosorption process there are many cracks (Fig. 1b) in the electrodeposited film due to the hydrogen insertion/removal into/from the bulk of the electrode. The results obtained in the Fig. 1c confirmed that the thickness of the electrodeposited layer is approximately $1 \mu \mathrm{m}$, since the values of the thickness calculated from the AAS measurements were in the range of approximately $0.8-1.1 \mu \mathrm{m}$.

XRD measurements revealed that in Pd-Ir alloys the lattice constant shrinks compared to Pd, which confirmed the classification of Pd-Ir alloys to the class of the contracted alloys (Fig. 2a, b).
The lattice constant for pure Pd equals to approximately $3.8907 \AA$; for pure Ir it is approximately $3.839 \AA$, whereas for Pd-Ir alloy, containing approximately $96 \%$ at. Pd it is approximately $3.885 \AA$. The value of lattice constant obtained for the Pd-Ir alloy is placed between the value of the pure metals (Fig. 2b), and can be a physicochemical evidence of the alloy formation.

In the case of the studied alloy negative deviation from Vegard's law is observed (the lattice is more contracted than expected). The composition calculated from Vegard's law (90.5\%) varies from the one obtained from AAS measurements (approximately 96\% Pd). The XRD analysis shows also the evolution of the diffraction patterns after electrode exposition under hydrogen (1 atm.) atmosphere. After interaction with hydrogen, the diffraction patterns from the hydrogen absorbing phase were shifted into lower values of scattering angle. In the case of the Pd-Ir alloy containing approximately $96 \%$ at. $\mathrm{Pd}$, the value of the lattice constant in hydride phase ( $\beta$-phase) is approximately $4.015 \AA$ and after changing the atmosphere into helium it achieves the previous value obtained before the exposition to hydrogen. According to La Prade et al. [23] the value of the lattice constant of Pd-Ir electrode (approximately $96 \% \mathrm{Pd}$ ) in the $\beta_{\min }$ phase equals to approximately $3.971 \AA$. Whereas in the case of the tested sample, lattice constant after absorption from the gas phase is approximately $4.015 \AA$. It indicates that the hydrogen absorbed in the tested sample is in the $\beta$-phase. The lattice constant expansion after hydrogen dissolution in Pd-Ir alloy (96\% at. Pd) is similar to the one obtained for pure $\mathrm{Pd}$ (the changes of the lattice constant: $\Delta \mathrm{a}_{\mathrm{Pd}}=0.134 \AA ; \Delta \mathrm{a}_{\mathrm{Pd}-\mathrm{II}}=0.130 \AA$ ). Irrespective of the atmosphere the width of the XRD diffraction patterns is stable which confirms the homogeneity of the hydrogen absorbing phase. In Fig. 2c, there is the SEM image of the Pd-Ir alloy electrode subjected to XRD analysis. It can be noticed that the exposure to gaseous hydrogen $(1 \mathrm{~atm}$.) also results in the cracks in the electrode volume, as was the case with electroabsorbed hydrogen.

\section{Electrochemical characterization - electrosorption of hydrogen}

Thin films of electrodeposited Pd-Ir alloys were subjected to a series of experiments involving electrochemical absorption of hydrogen from the acidic medium. Before the main measurement, each of the Pd-Ir alloy electrode was polarized in the socalled "hydrogen region" (potential range: $-0.1 \div 0.3 \mathrm{~V}$ ), to obtain repetitive results. This approach, called hydrogen pretreatment procedure (HPP), was previously used for Pd and Pd-based electrodes in aqueous and non-aqueous electrolytes $[4,5,9]$. Figure 3 a shows cyclic voltammetry behavior of PdIr alloys of various compositions after HPP. It is clearly visible that Pd alloying with Ir significantly influences on the position of hydrogen desorption signal $\left(E_{\mathrm{p}}{ }^{\text {an }}\right)$. This effect is already 

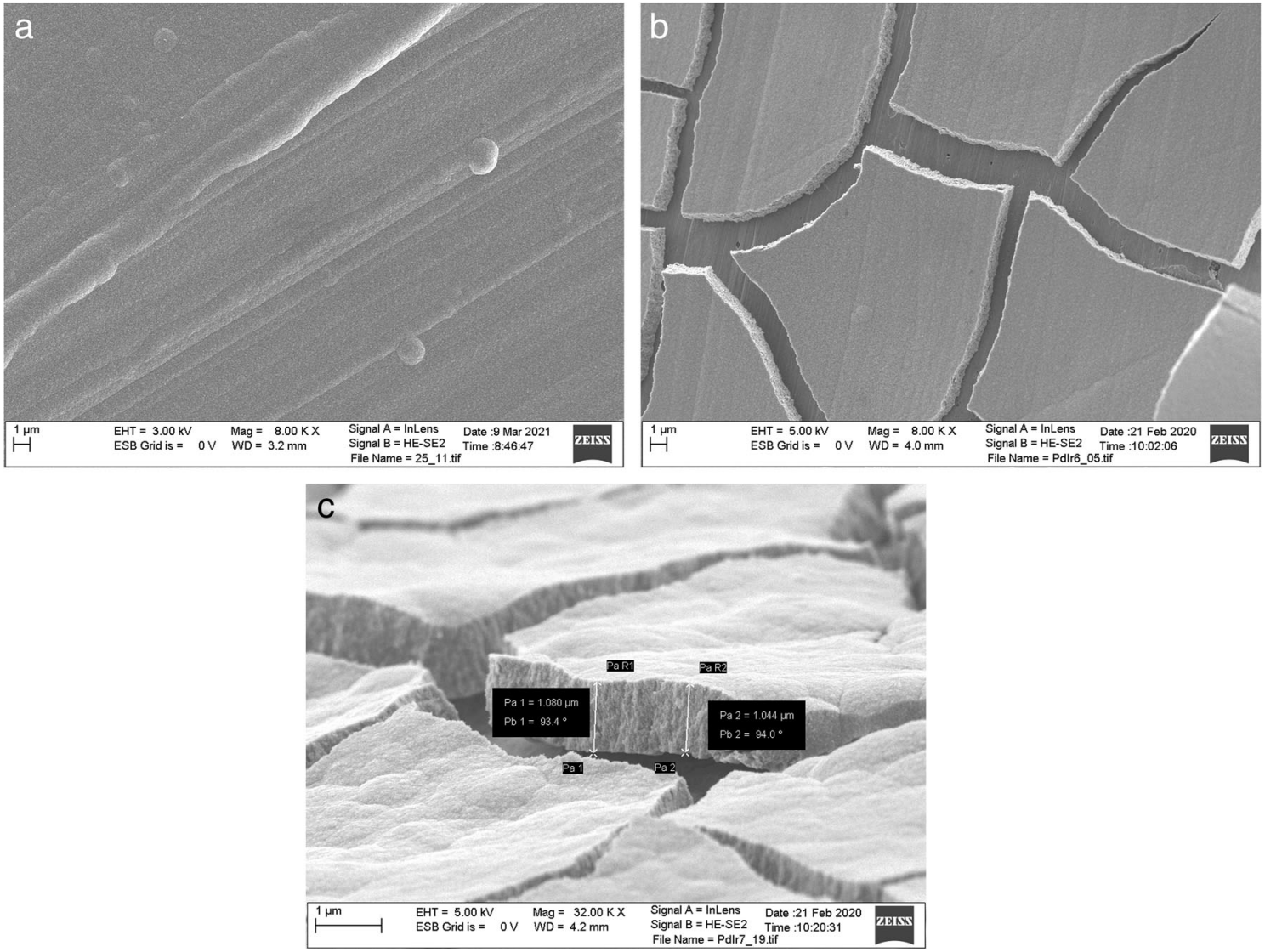

Fig. 1 a SEM image of the Pd-Ir electrode (approximately 97\% Pd, EDS). b SEM image of the Pd-Ir electrode (97\% Pd, EDS) after hydrogen EDS) after hydrogen electrosorption. The segments with arrows indicate electrosorption. c SEM image of the Pd-Ir electrode (approximately 99\%,

the thickness of the electrodeposited layer

Fig. 2 a XRD patterns of Pd-Ir alloy (approximately $96 \% \mathrm{Pd}$ (EDS) in the bulk) in helium and hydrogen atmosphere. $\mathbf{b}$ The evolution of 220 XRD signal in different atmospheres. c SEM image of the Pd-Ir electrode subjected to XRD analysis
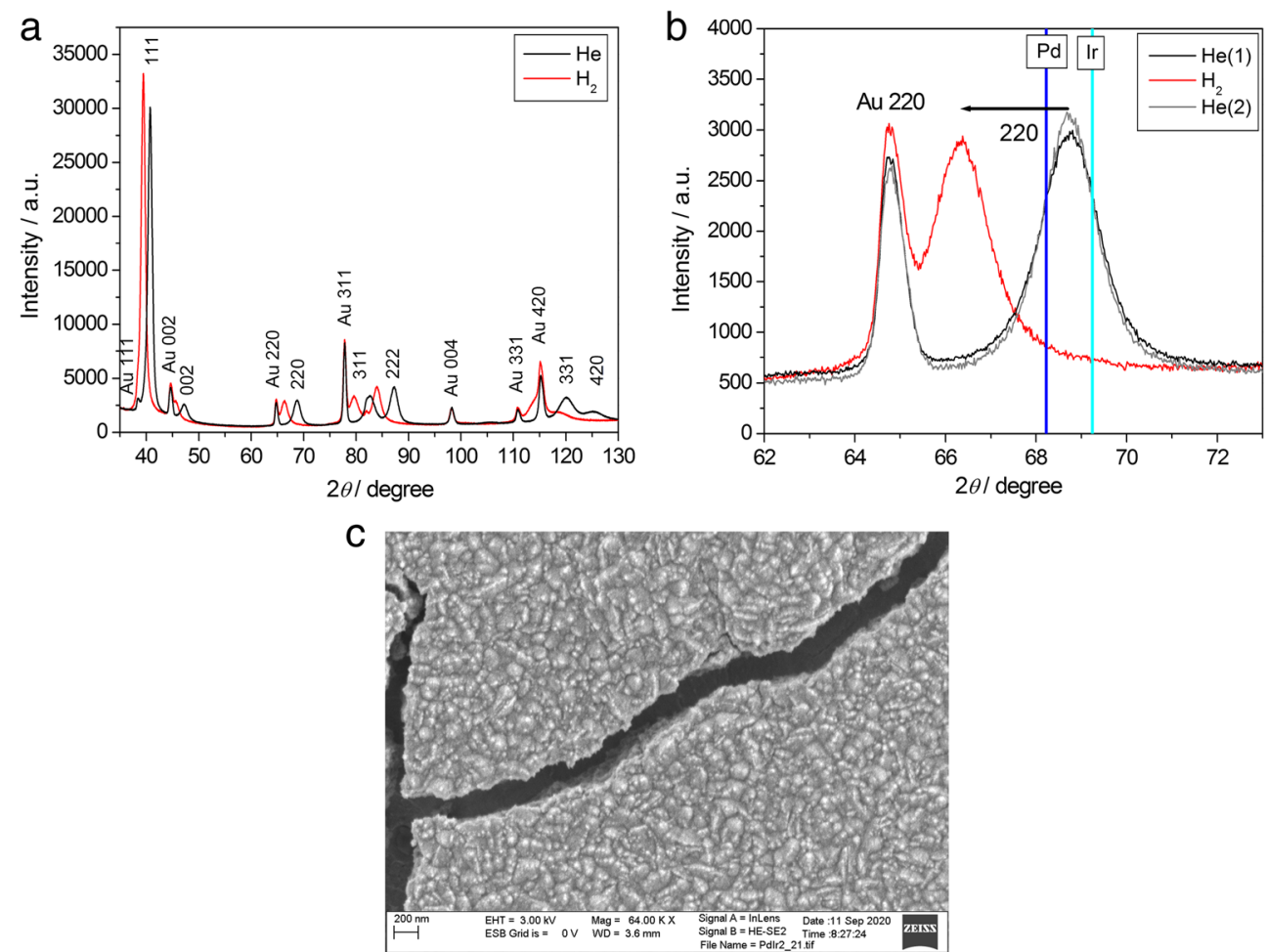

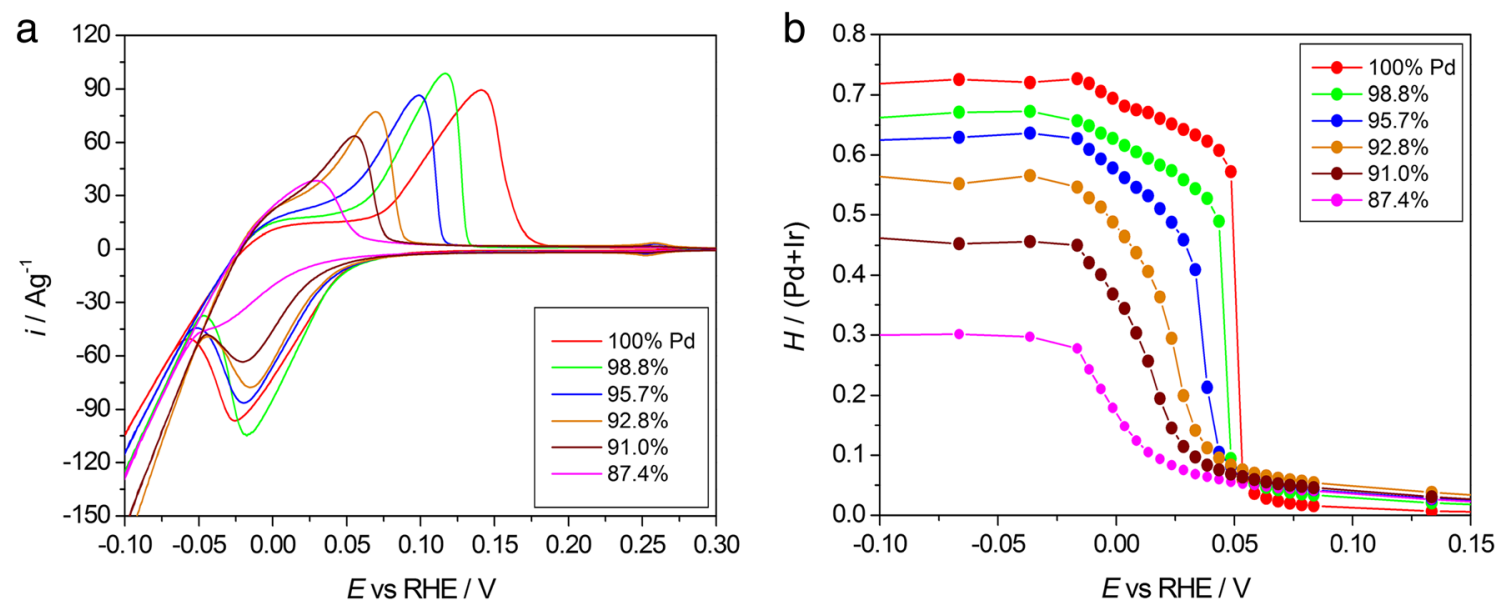

Fig. 3 a Cyclic voltammetry behavior of selected compositions of Pd-Ir alloys in $0.5 \mathrm{M} \mathrm{H}_{2} \mathrm{SO}_{4}$; scan rate: $0.01 \mathrm{Vs}^{-1}$. b Hydrogen electrosorption isotherms (HEIs) of Pd-Ir alloys

evident for an alloy containing approximately $1.2 \%$ Ir. The value of $E_{\mathrm{p}}{ }^{\text {an }}$ decreases with the increasing Ir content which indicates the facilitation of the process of hydrogen removal. Ir addition to Pd results also in slight changes in the position of the hydrogen absorption signals $\left(E_{\mathrm{p}}{ }^{\mathrm{ca}}\right)$. For the alloys able to the formation of the hydride phase $E_{\mathrm{p}}{ }^{\mathrm{ca}}$ is higher than in Pd. The decreasing difference between $E_{\mathrm{p}}{ }^{\mathrm{an}}$ and $E_{\mathrm{p}}{ }^{\mathrm{ca}}$ indicates that the presence of Ir increases the electrochemical reversibility of the process of hydrogen absorption. Furthermore, in the potential range $-0.1 \div 0.2 \mathrm{~V}$ there are only single signals originating from the hydrogen insertion/removal into/from the bulk of Pd-Ir electrodes. It is an electrochemical confirmation that there is only one Pd-rich hydrogen absorbing phase.

Hydrogen electrosorption isotherms (HEIs) of the same samples as presented in Fig. 3a are shown in the Fig. 3b. In the case of Pd-Ir alloys the shapes of electrosorption isotherms are similar (S-shape) than that obtained for Pd and Pd-based alloys in different electrolytes [3-5, 9]. In the HEI three main parts can be distinguished: (1) first plateau assigned to the hydrogen absorbed in the $\alpha$-phase (solid solution of hydrogen in the alloy) and hydrogen adsorbed, (2) the abrupt increase in the values of $\mathrm{H} /(\mathrm{Pd}+\mathrm{Ir})$ corresponding to $\alpha \rightarrow \beta$ phase transition, and (3) second plateau assigned to hydrogen absorbed in the $\beta$-phase (hydride) [33]. In the contracted alloys the potential of the $\alpha \rightarrow \beta$ phase transition decreases with increasing amount of the alloying metals $[9,11,12]$. It is due to the fact that in contracted alloys greater work than in pure $\mathrm{Pd}$ is needed for lattice expansion during hydrogen absorption. This behavior is also observed for examined Pd-Ir alloys and constitutes the electrochemical confirmation that Pd alloying with Ir leads to the creation of contracted alloys. The increase of the Ir content in the alloy influences on the decrease in the $\alpha \rightarrow \beta$ phase transition potential (see Figs. $3 b$ and 4).

The relation between $\alpha \rightarrow \beta$ phase transition potential $\left(E_{\alpha \rightarrow}\right.$ $\beta$ ) and alloy bulk composition exhibits linear correlation (see
Fig. 4). This behavior was also noticed for other contracted/ pseudo-contracted binary Pd-based alloys such as Pd-Rh, Pd$\mathrm{Ru}$, and Pd-Pt [9]. Also, in the case of Pd-Ir alloys electrochemical measurements leading to the determination of the $E_{\alpha \rightarrow \beta}$ can serve as a non-destructive method of the estimation of the alloy bulk compositions. According to the course of the $E_{\alpha \rightarrow \beta}$ versus Pd content, the Pd-Ir alloys exhibit intermediate behavior between $\mathrm{Pd}-\mathrm{Pt} / \mathrm{Pd}-\mathrm{Rh}$ and $\mathrm{Pd}-\mathrm{Ru}$ alloys (in the case of Pd-Pt and Pd-Rh alloys the relations of $E_{\alpha \rightarrow \beta}$ vs Pd content have similar courses). However, the course of $E_{\alpha \rightarrow \beta}$ versus $\mathrm{Pd}$ content for Pd-Ir alloys reflects more the one for Pd-Ru alloys [9].

Alloying Pd with Ir results also in the changes in the maximum hydrogen absorption capacity. One can notice in Fig. 5 that for all the tested compositions of the Pd-Ir alloys the hydrogen absorption capacity is lower than for pure Pd. For

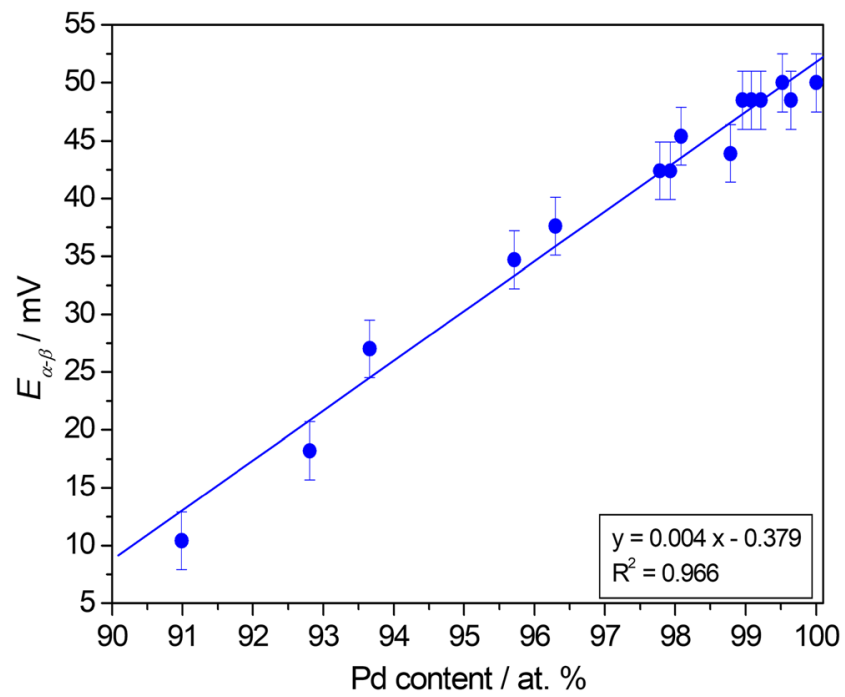

Fig. 4 The dependence of the $\alpha \rightarrow \beta$ phase transition potential on the PdIr alloy compositions 


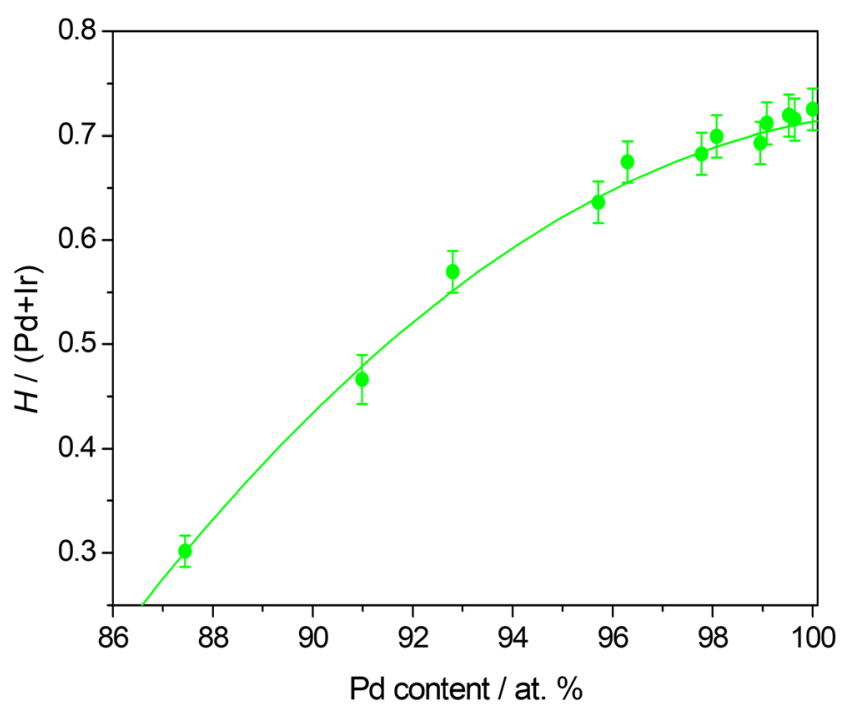

Fig. 5 The influence of the Pd-Ir alloy compositions on the maximum hydrogen capacity $(H / \mathrm{Pd}+\mathrm{Ir})$

the alloys containing less than $3 \%$ at. of $\mathrm{Ir}, H /(\mathrm{Pd}+\mathrm{Ir})$ is very close to the $H / \mathrm{Pd}(0.72)$, exceeding $0.66 \pm 0.02$. For this group of Pd-Ir alloys (Ir content below 3\% at.) hydrogen absorption capacity of Pd-Ir alloys is similar to the one obtained for Pd-Pt alloys. With the increase of the Ir content, the decrease in the hydrogen absorption capacity is more abrupt, and more reflects the behavior of the Pd-Ru alloys. Theoretical predictions involving calculations of hydrogen absorption energy and density of states made by Yayama et al. [34] indicate that there was huge possibility of the increased hydrogen absorption capacity, similarly as in the case of Pd-Rh alloys. However, the values of the hydrogen absorption capacity obtained electrochemically for a wide range of Pd-Ir alloy compositions, contradict cited hypothesis. Interestingly, examined system of Pd-Ir alloys exhibits hydrogen electrosorption properties closer to Pd-Pt and Pd-Ru alloys rather than Pd-Rh alloys, as it has been predicted from the similarities of electronic structures.

Our further studies on the Pd-Ir-H system will involve i.a. the detailed examination of hydrogen electrosorption properties in different electrolytes, kinetics, and thermodynamics of hydride phase formation. Pd-Ir alloys will be also subjected to thorough physicochemical characterization.

\section{Conclusions}

The potentiostatic electrodeposition from chloride baths containing $\mathrm{Pd}(\mathrm{II})$ and $\operatorname{Ir}(\mathrm{III})$ ions enables to obtain compact and not cracked Pd-Ir (above $87 \%$ at. Pd) thin films on a gold matrix. The formation of the contracted alloys and their homogeneity were confirmed electrochemically and by XRD analysis. The process of hydrogen oxidation is facilitated in Pd-Ir alloys (compared to Pd). Already a little addition of Ir (below $2 \%$ at.) leads to the significant increase of reversibility of the process of hydrogen electrosorption. Pd alloying with Ir results in the decrease in the $\alpha \rightarrow \beta$ phase transition potential (typical for contracted alloys) and the decrease in the hydrogen absorption capacity. The variations of the $\alpha \rightarrow \beta$ phase transition potentials and hydrogen absorption capacities with $\mathrm{Pd}$ content in Pd-Ir alloys reflect the intermediate behavior between Pd-Pt and Pd-Ru alloys. The theoretical predictions indicating the possibility of higher than in Pd hydrogen absorption capacities have not been confirmed.

Open Access This article is licensed under a Creative Commons Attribution 4.0 International License, which permits use, sharing, adaptation, distribution and reproduction in any medium or format, as long as you give appropriate credit to the original author(s) and the source, provide a link to the Creative Commons licence, and indicate if changes were made. The images or other third party material in this article are included in the article's Creative Commons licence, unless indicated otherwise in a credit line to the material. If material is not included in the article's Creative Commons licence and your intended use is not permitted by statutory regulation or exceeds the permitted use, you will need to obtain permission directly from the copyright holder. To view a copy of this licence, visit http://creativecommons.org/licenses/by/4.0/.

\section{References}

1. Flanagan TB, Lewis FA (1959) Hydrogen absorption by palladium in aqueous solution. Trans Faraday Soc 55:1400-1408

2. Lewis FA (1967) The palladium hydrogen system. Academic Press, London and New York

3. Czerwiński A, Marassi R, Zamponi S (1991) The absorption of hydrogen and deuterium in thin palladium electrodes. Part I. Acidic solutions. J Electroanal Chem 316:211-221

4. Pająk M, Hubkowska K, Czerwiński A (2018) The study of hydrogen sorption in palladium limited volume electrode from DEMATFO ionic liquid. J Electroanal Chem 825:73-76

5. Hubkowska K, Soszko M, Symonowicz M, Łukaszewski M, Czerwiński A (2017) Electrochemical behavior of a Pd thin film electrode in concentrated alkaline media. Electrocat 8(4):295-300

6. Zalineeva A, Baranton S, Coutanceau C, Jerkiewicz G (2015) Electrochemical behavior of unsupported shaped palladium nanoparticles. Langmuir 31(5):1605-1609

7. Yang N, Yee JK, Zhang Z, Kurmanaeva L, Cappillino P, Stavila V, Lavernia EJ, San Marchi C (2015) Hydrogen sorption characteristics of nanostructured $\mathrm{Pd}-10 \mathrm{Rh}$ processed by cryomilling. Acta Mater 82:41-50

8. Comisso N, De Ninno A, Del Giudice E, Mengoli G, Soldan P (2004) Electrolytic hydriding of Pd79.5Rh20.5 alloy. Electrochim Acta 49(9-10):1379-1388

9. Hubkowska K, Czerwiński A (2020) Tuning hydrogen sorption properties of Pd by its alloying with $\mathrm{Ru}, \mathrm{Rh}$, and Pt: the study of binary alloys in concentrated alkaline media. J Solid State Electrochem 24(11-12):3135-3143

10. Ferrari P, Diaz-Droguett DE, Rojas S, Cabrera AL (2014) Inhibition of hydrogen absorption in bulk Pd by the formation of Ru-Pd surface alloy. Thin Solid Films 550:732-737

11. Hubkowska K, Łukaszewski M, Czerwiński A (2014) Thermodynamics of hydride formation and decomposition in 
electrodeposited Pd-rich Pd-Ru alloys. Electrochem Commun 48: $40-43$

12. Hubkowska K, Łukaszewski M, Koss U, Czerwiński A (2014) Characterization and electrochemical behavior of Pd-rich Pd-Ru alloys. Electrochim Acta 132:214-222

13. Tayal A, Seo O, Kim J, Kumara LSR, Song C, Hiroi S, Chen Y, Kobayashi H, Kitagawa H, Sakata O (2019) Effects of interfacial structure of Pd-Pt nanoparticles on hydrogen solubility. J Alloys Compd 791:1263-1269

14. Deepti KH, Tripathic A, Deyd AB, Gupta M, Krishna R, Avasthi DK (2019) Improved hydrogen sensing behaviour in ion-irradiated Pd-Au alloy thin films. Sensors Actuators B Chem 301:127006

15. Sun L, Chen M, Peng X, Xie B, Han M (2016) The effects of Ni contents on hydrogen sensing response of closely spaced Pd-Ni alloy nanoparticle films. Int J Hydrog Energ 41(2):1341-1347

16. Rosamilia JM, Abys JA, Miller B (1991) Electrochemical hydrogen insertion into palladium and palladium-nickel thin films. Electrochim Acta 36:1203-1208

17. Zhao M, Brouwer JC, Sloof WG, Bottger AJ (2020) Surface segregation of $\mathrm{Pd}-\mathrm{Cu}$ alloy in various gas atmospheres. Int J Hydrog Energ 45(41):21567-21572

18. Tang J, Yamamoto S, Koitaya T, Yoshikura Y, Mukai K, Yoshimoto S, Matsuda I, Yoshinobu J (2019) Hydrogen adsorption and absorption on a Pd-Ag alloy surface studied using in-situ X-ray photoelectron spectroscopy under ultrahigh vacuum and ambient pressure. Appl Surf Sci 463:1161-1167

19. Tripathi SN, Bharadwaj SR, Chandrasekharaiah MS (1991) The IrPd (iridium-palladium) system. J Phase Equilibria 12(5):603-605

20. Raub E, Plate W (1957) The palladium-iridium alloys. Z Metallkd 48:444-447

21. Woods R (1976) Chemisorption at electrodes: hydrogen and oxygen on noble metals and their alloys. In: Bard AJ (ed) Electroanalytical Chemistry. Marcel Dekker, New York

22. Woods R (1974) Hydrogen adsorption on platinum, iridium and rhodium electrodes at reduced temperatures and the determination of real surface area. J Electroanal Chem 49(2):217-226

23. LaPrade M, Allard KD, Lynch JF, Flanagan TB (1974) Absorption of hydrogen by iridium/palladium substitutional alloys. J Chem Soc Faraday Trans 170:1615-1630

24. Akamaru S, Hara M, Matsuyama M (2014) Alloying effects on the hydrogen-storage capability of Pd-TM-H $(\mathrm{TM}=\mathrm{Cu}, \mathrm{Au}, \mathrm{Pt}, \mathrm{Ir})$ systems. J Alloys Compd 614:238-243
25. Zlotea C, Morfin F, Nguyen TS, Nguyen NT, Nelayah J, Ricolleau C, Latroche M, Piccolo L (2014) Nanoalloying bulk-immiscible iridium and palladium inhibits hydride formation and promotes catalytic performances. Nanoscale 6(17):9955-9959

26. Malouche A, Oumellal Y, Ghimbeu CM, de Yuso AM, Zlotea C (2017) Exploring the hydrogen absorption into Pd-Ir nanoalloys supported on carbon. J Nanopart Res 19(8):270-280

27. Kobayashi H, Yamauchi M, Ikeda R, Yamamoto T, Matsumurade S, Kitagawa H (2018) Double enhancement of hydrogen storage capacity of Pd nanoparticles by 20 at\% replacement with Ir; systematic control of hydrogen storage in $\mathrm{Pd}-\mathrm{M}$ nanoparticles $(\mathrm{M}=\mathrm{Ir}$, Pt, Au). Chem Sci 9(25):5536-5540

28. Wicke E, Frolich K (1989) Electronic and elastic effects in the phase diagrams of binary Pd alloy hydrides. Z Phys Chem N F 163(Part_1):35-40

29. Yang T, Ma Y, Huang Q, Cao G, Wan S, Li N, Zhao H, Sun X, Yin F (2015) Palladium-iridium nanowires for enhancement of electrocatalytic activity towards oxygen reduction reaction. Electrochem Commun 59:95-99

30. Allerston LK, Hodgson D, Gibbs C, Brett DJL, Rees NV (2020) Increased stability of palladium-iridium-gold electrocatalyst for the hydrogen oxidation reaction in polymer electrolyte membrane fuel cells. Electroanalysis 32(12):2893-2901

31. Șen B, Aygün A, Șavk A, Akocak S, Șen F (2018) Bimetallic palladium-iridium alloy nanoparticles as highly efficient and stable catalyst for the hydrogen evolution reaction. Int J Hydrog Energy 43(44):20,183-20,191

32. Ziaei-Azad H, Semagina N (2016) Iridium addition enhances hydrodesulfurization selectivity in 4,6-dimethyldibenzothiophene conversion on palladium. Appl Catal B Environ 191:138-146

33. Łukaszewski M, Hubkowska K, Koss U, Czerwiński A (2013) On the nature of voltammetric signals originating from hydrogen electrosorption into palladium-noble metal alloys. Materials 6(10): 4817-4835

34. Yayama T, Ishimoto T, Koyama M (2015) Effect of alloying elements on hydrogen absorption properties of palladium-based solid solution alloys. J Alloys Compd 653:444-452

Publisher's note Springer Nature remains neutral with regard to jurisdictional claims in published maps and institutional affiliations. 